\title{
State Estimation based Inverse Dynamic Controller for Hybrid system using Artificial Neural Network
}

\author{
Xiao Laui ${ }^{1}$, Rui-Lain Chua ${ }^{2}$, \\ ${ }^{I}$ Research Scholar, University of Hong Kong, China, xiaolauilaui@gmail.com \\ ${ }^{2}$ Professor, University of Hong Kong, China. \\ *Correspondence: Xiao Laui, University of Hong Kong, China, xiaolauilaui@gmail.com
}

\begin{abstract}
State Estimation Based Inverse Dynamics Controller (SEBIDC), which utilizes an Artificial Neural Network (ANN) based state estimation scheme for nonlinear autonomous hybrid systems which are subjected to state disturbances and measurement noises that are stochastic in nature. A salient feature of the proposed scheme is that it offers better state estimates and hence a better control of non-measurable state variables with a nonlinear approach in correcting the a priori estimates by avoiding statistical linearization involved in existing approaches based on derivative free estimation methods. Simulation results guarantees significant reduction in Integral Square Error (ISE) and standard deviation ( $\sigma$ ) of error, between the controlled variable and set point and control signal computation time when compared with best existing related work based on Unscented Kalman Filter (UKF) and Ensemble Kalman Filter (EnKF). Detailed analysis of the experimental results on real plant under different operating conditions such as servo and regulatory operations, initial condition mismatch, and different types of faults in the system, confirms robustness of proposed approach in these conditions and support the simulation results obtained. The main advantage of the proposed controller is that the control signal computation time is very much less than the selected sampling time of the process, so direct control of the plant is possible with this approach.
\end{abstract}

Keywords: Artificial Neural Network, Hybrid Dynamic Systems, State Estimation, Inverse Dynamics Controller.

\section{INTRODUCTION}

Systems in which it may be required to model inherent process discontinuities, where the continuous behavior is drastically changed, or use actuators and sensors which are often fundamentally discontinuous, or use discrete events that can be a useful abstraction to model various mode switching used in the specification and control of the basically continuous process, require hybrid systems modeling and control approach ([1], [2] , [3] and [4]). Kalman filter (KF) [5] and extended Kalman filter (EKF) [6] are used as a state estimator in conventional state observers. For linear systems as uncertainties in state and measurement equations can be modeled as Gaussian white noise processes Kalman filter can generate optimal estimates of state. Extended Kalman filter (EKF), which is a natural extension of the linear filter to the nonlinear domain through analytical linearization, can be used in state estimation of nonlinear systems.

\section{ARTICLE INFORMATION}

Author(s): Xiao Laui;

Received: 13 March 2020; Revision: 18 March 2020; Published: 10 April 2020;

e-ISSN: 2347-470X;

Paper Id:IJEER-080102;

Citation: http://doi.org/10.37391/ijeer.080102

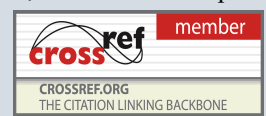

Webpage-link: http://ijeer.forexjournal.co.in/archive/volume-8/ijeer080102.html
In this approach, the estimated states are obtained by using Taylor series expansion of the nonlinear state transition operator. But, it requires analytical computation of Jacobians at each time step, which is considered to be computationally demanding for complex nonlinear systems. Also it is required that nonlinear function vector appearing in state dynamics and output dynamics should be smooth and at least once differentiable. However, dynamical models of Hybrid systems involve discontinuities due to switching of the discrete state values. As Jacobians cannot be computed for non-smooth functions, EKF cannot be realized in Hybrid systems [7]. In [8] a moving horizon based state estimation approach has been reported for hybrid system estimation. But, the use of fixed arrival cost used in the moving horizon estimator formulation result in sub-optimal state estimates. The authors in [9] have proposed UKF as an alternative to EKF so that the main shortcoming of EKF when used for highly nonlinear system is eliminated. [10] has proposed a derivative free nonlinear filtering technique for nonlinear hybrid systems. As far as the control element is concerned, [11] had proposed a robust model predictive control (RMPC) scheme for a class of hybrid system such as piece wise affine system to ensure simple and fast suboptimal solution for the control problem with reduced computation time. Similarly, the nonlinear model predictive control (NMPC) in [7] and [12], and fault tolerant model 
predictive control in [13], for hybrid systems, used UKF approach in its state estimation part. All the state estimation schemes for hybrid systems in literature involve analytical or statistical linearization [14] and preclude their use from systems which require more accurate state estimates. Introduction of Artificial Neural Network (ANN) in state estimation and control of different systems considerably improves the performance which is very clear from the works reported in [15], [16], [17] and [18]. But, in the case of [15], if a nonlinear scheme is used in correction of a priori estimates, more accurate state estimates can be obtained in hybrid systems also and this has been implemented in this work. The distinguished feature of the proposed approach is that it uses nonlinear ANN to correct the a priori estimates and gives better estimate than its counterparts. Once trained for sufficient variety of input data, including ill-conditioned system data, the ANN-based state estimator provides accurate estimates of the system states. Further, an ANN based Controller (ANNC), using this estimator, is developed for controlling the non-measurable states of the hybrid system. The proposed controller (ANNC) provides better performance and has following advantages over existing schemes.

- Apart from analytical and statistical linearization in the correction part of EKF and UKF based controllers respectively; proposed controller uses a nonlinear correction approach using ANN to correct the a priori estimates and hence offers a better state estimates by avoiding the linearization. The correction part of ANNC is completely parameter independent, and thereby gives better state estimates even under there is mismatch in the parameters.

- ANN has built in noise rejection capability which makes the ANNC scheme robust in performance.

Comparative analysis of the proposed approach with best relating work based on UKF (statistical linearization approach) and EnKF (Particle Filter) is made in terms of ISE in state estimate on the same benchmark model and it reveals that the proposed approach is able to reduce the error in state estimates. Detailed performance evaluation of the proposed approach under servo - regulatory operations and plant model parameter mismatch were conducted. Detailed analysis of the experimental results on the real plant under different operating conditions such as initial condition mismatch, and different types of faults in the system confirms efficacy of proposed approach. Here, an inverse dynamics controller is utilized for controlling the non-measurable states of the system so that the computational burden is very much reduced when compared with the model predictive control scheme implemented in [7], [12] and [13] without compromising the performance. Also the constraints handling capability for this scheme is also achieved with this approach by introducing upper and lower limiting functions at the output of both estimator and controller. The rest of the paper is organized as follows. In section 2, description of the ANN state estimation algorithm developed for hybrid systems is given. The Section 3 explains the SEBIDC scheme. Simulation results and detailed performance analysis of the proposed scheme and with comparison to the best related work is given in section 4. Experimental results and its analysis are presented in Section 5. Finally, section 6 summarizes the paper.

\section{ANN based Hybrid State Estimation}

In this scheme, an ANN based correction is developed. As in the case of EKF and UKF, ANN based state estimation is also recursive in nature. Even though it has the same framework of Kalman filter based state estimator, it is designed for eliminating the analytical and statistical linearization [14] used in the case of EKF and UKF. This structure is suggested because recurrent type of ANN is better for the complex dynamic system [18]. The schematic diagram of proposed ANNC is as given in Fig. 1.

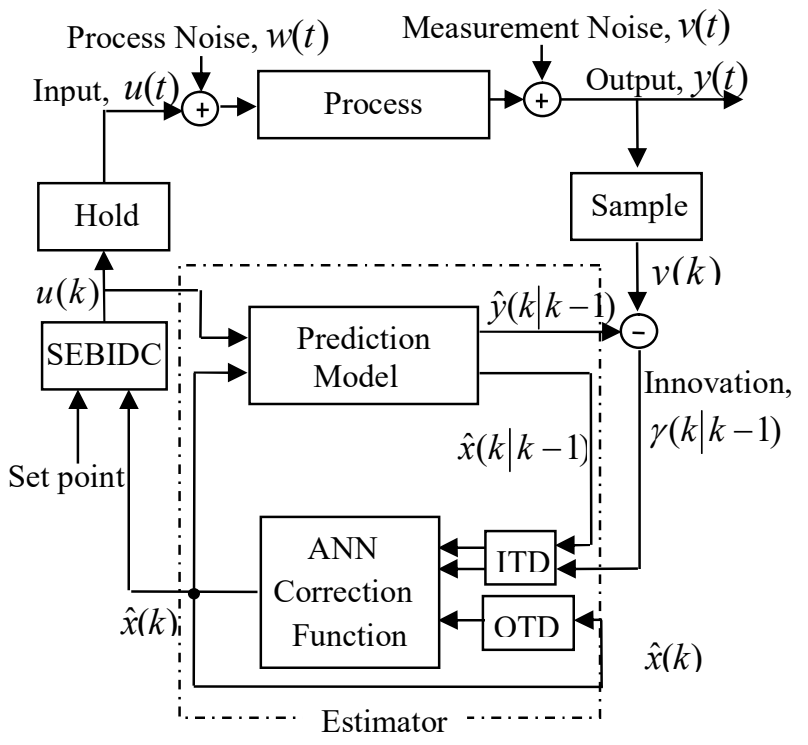

Fig.1. Schematic representation of proposed ANNC

The detailed nonlinear auto regressive with exogenous input (NARX) structure used for the considered problem is given in Fig. 2 and the other NARX parameters used for this study are provided in Table 1.

The current output can be predicted as a function of present and past inputs and past outputs as given below, in which $\mathrm{Y}$ and $\mathrm{X}$ represent the outputs and inputs of the network respectively and $\mathrm{K}_{\mathrm{NN}}$ is a nonlinear ANN function.

$\mathrm{Y}(\mathrm{k})=\mathrm{K}_{\mathrm{NN}}\{\mathrm{X}(\mathrm{k}), \mathrm{X}(\mathrm{k}-\mathrm{m}), \mathrm{Y}(\mathrm{k}-1) \ldots \mathrm{Y}(\mathrm{k}-\mathrm{n})\}$ 
Table 1: ANN Parameters

\begin{tabular}{ll}
\hline Parameter & Value \\
\hline ANN Structure & NARX \\
No. of hidden layers & 1 \\
Hidden Layer neurons & 5 \\
Hidden layer activation function & 'tan sigmoid' \\
Output layer activation function & 'purelin' \\
No of epochs & 100 \\
No of exogenous inputs & 4 \\
No. of delayed inputs & 0 \\
No of outputs & 3 \\
No. of feedback output delays & 2 \\
Training method & Back propagation \\
Training function & Levenberg-Marquardt \\
Performance Function & Mean Square Error \\
\hline
\end{tabular}

A sequence of current and past input vectors $(\mathrm{X}(\mathrm{k}), \mathrm{X}(\mathrm{k}-1), \mathrm{X}$ $(\mathrm{k}-\mathrm{m})$ ) are obtained by passing $\mathrm{X}(\mathrm{k})$ through an input time delay unit, ITD (0: $\mathrm{m})$. Similarly output time delay unit, OTD (1: n) provides a sequence of past output vectors (Y (k-1), Y $(\mathrm{k}-\mathrm{n}))$. For the considered problem, the input and the output are $\mathrm{X}(k)=\left[\hat{h}_{1}(k \mid k-1), \hat{h}_{2}(k \mid k-1), \hat{h}_{3}(k \mid k-1), \gamma(k \mid k-1)\right]^{\mathrm{T}}$ $\mathrm{Y}(k)=\left[\hat{h}_{1}(k), \hat{h}_{2}(k), \hat{h}_{3}(k)\right]^{\mathrm{T}}$ respectively.

Similar to Kalman filter based state estimators and its nonlinear extensions, the proper value for the initial state vector is assumed for the prediction model. The input and output measurements are made from the process and the input measurement are presented to the state prediction model (F) along with the assumed initial state vector in order to compute the time updated values for states.

$\hat{x}(k \mid k-1)=\mathrm{F}(\hat{x}(k-1), u(k))$

With, $\hat{x}(k-1)=\hat{x}(0)=E[x(0)]$, the assumed initial value of state vector.

The a priori state estimates, $\hat{x}(k \mid k-1)$ can be given to the output model $(\mathrm{H})$ so that a priori estimates of the output, $\hat{y}(k \mid k-1)$ can be obtained as

$\hat{y}(k \mid k-1)=\mathrm{H}[\hat{x}(k \mid k-1)]$

The innovation between plant output $y(k)$ and a priori output estimate $\hat{y}(k \mid k-1)$ is calculated as

$\gamma(k \mid k-1)=y(k)-\hat{y}(k \mid k-1)$
In the correction step of the algorithm, the a priori state estimates will be corrected using this innovation with the help of the ANN to obtain a posteriori estimates of state vector $\hat{x}(k \mid k)$.

$\hat{x}(k)=\mathrm{K}_{\mathrm{NN}}\{\operatorname{ITD}(\hat{x}(k \mid k-1), \gamma(k \mid k-1)), \mathrm{OTD}(\hat{x}(k))\}$

These estimated states are fed back to the controller for calculating the new input signal to the plant.

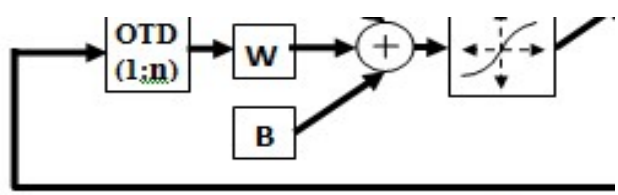

(a)

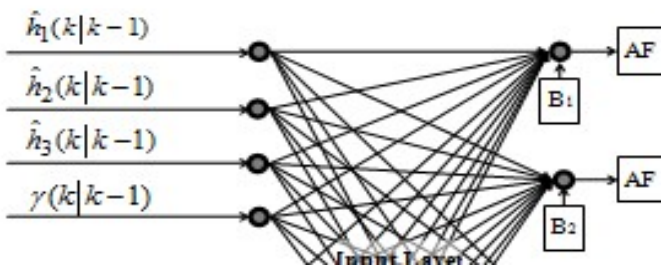

Figure 2: NARX structure for the three-tank hybrid system

\section{State Estimation Based Inverse Dynamics Controller (SEBIDC)}

In hybrid systems, for the controller design, its mode switching property has also to be taken in to account. For such processes, model based control schemes are proposed in the literature ([12], [13], [19], [20], [21] and [22]) for obtaining the satisfactory control of the output variables. In this work a model based control namely inverse dynamics controller (IDC) have been implemented for controlling the nonmeasurable states of Hybrid System. The objective of this section is to review the non-linear dynamic control technique that can be applied to develop a non-measurable level control system that is valid over the entire operating region of the hybrid three-tank system which is described below.

Consider the nonlinear system of the form,

$\dot{x}=A(x)+B(x) u$

$y=C x$

Where, $A(x)=(n \times 1)$ vector, $\mathrm{B}(x)=(n \times m)$ matrix, $\mathrm{C}=(3 \rightarrow) \times$ 1) vector.

Using the inverse dynamics of (6) and (7), input vector $u$ can be represented as a function of $v$ and $x$,

Let it be $u=\mathrm{F}_{1}(v, x)$ 
Where, $\mathrm{F}_{1}$ is a nonlinear function and $v$ is the input to the inverse system.

Implementation of the inverse dynamics controller of (6) and (7) is done by repeatedly differentiating the measurement function until the input variable $u$ appears.

Differentiating (7)

$\dot{y}=C \dot{x}$

$\dot{y}=C[A(x)+B(x) \cdot u]$

$\dot{y}=A^{*}(x)+B^{*}(x) \cdot u$

Where, $A^{*}(x)=C \cdot A(x)$ and $B^{*}(x)=B \cdot A(x)$

So the control law $u$ can be written as per [20] as

$u=B^{*-1}(x)\left[v-A^{*}(x)\right]$

A sufficient condition for the existence of an inverse system model to (6) and (7) is that $B^{*}$ in (11) be non-singular. If this is the case, then the inverse system model takes the form,

$\dot{x}=A(x)+B(x)[-F(x)+G(x) v]$

Where, $F(x)=B^{*^{-1}}(x) A^{*}(x)$ and $G(x)=B^{*^{-1}}(x)$

$$
\dot{x}=A(x)+B(x)[-F(x)+G(x) v]
$$

The input to the inverse system is $v=y-y_{\text {ref }}$

As the hybrid three-tank system, considered under this study can be directly represented in the form of (6) and (7), applying this procedure will yield the control law as

$$
\begin{aligned}
& F_{i n_{1}}=\mathrm{A}_{1} \mathrm{C}_{1}\left(\mathrm{~h}_{1 \mathrm{sp}}-h_{1}\right)+Q_{1}+Q_{3}+Q_{5} \\
& F_{i n_{2}}=\mathrm{A}_{2} \mathrm{C}_{2}\left(\mathrm{~h}_{2 \mathrm{sp}}-h_{2}\right)+Q_{2}+Q_{4}+Q_{7}
\end{aligned}
$$

Since $h_{1}, h_{2}, Q_{1}, Q_{2}, Q_{3}, Q_{4}, Q_{5}$, and $Q_{7}$, are non-measureable for the considered problem, the estimated values can be used so that the controller becomes an estimator based inverse dynamics controller.

$$
\begin{aligned}
& F_{i n_{1}}=\mathrm{A}_{1} \mathrm{C}_{1}\left(\mathrm{~h}_{1 \mathrm{sp}}-\hat{h}_{1}\right)+\hat{Q}_{1}+\hat{Q}_{3}+\hat{Q}_{5} \\
& F_{i n_{2}}=\mathrm{A}_{2} \mathrm{C}_{2}\left(\mathrm{~h}_{2 \mathrm{sp}}-\hat{h}_{2}\right)+\hat{Q}_{2}+\hat{Q}_{4}+\hat{Q}_{7}
\end{aligned}
$$

Where, $\mathrm{C}_{1}$ and $\mathrm{C}_{2}$ are controller tuning parameter, and its values are varied from $0<\mathrm{C} 1, \mathrm{C} 2<1$ on separate runs and the integral square error between the controlled variable and the set point, is noted. The values of $\mathrm{C}_{1}$ and $\mathrm{C}_{2}$, which give the minimum ISE, are selected as the tuning parameters. The $\mathrm{h}_{1 \mathrm{sp}}$ and $\mathrm{h}_{2 \text { sp }}$ are the corresponding desired values of water levels.

\section{Simulation Results and Performance}

\section{Analysis}

The schematic representation of hybrid three-tank system is given in Fig.3. The benchmark system used in [7] is used here with same levels in three tanks $\left(h_{1}, h_{2}\right.$ and $\left.h_{3}\right)$ as continuous states and $z_{1}$ and $z_{2}$ variables as discrete states for evaluating the performance the controller in comparison to best related work.

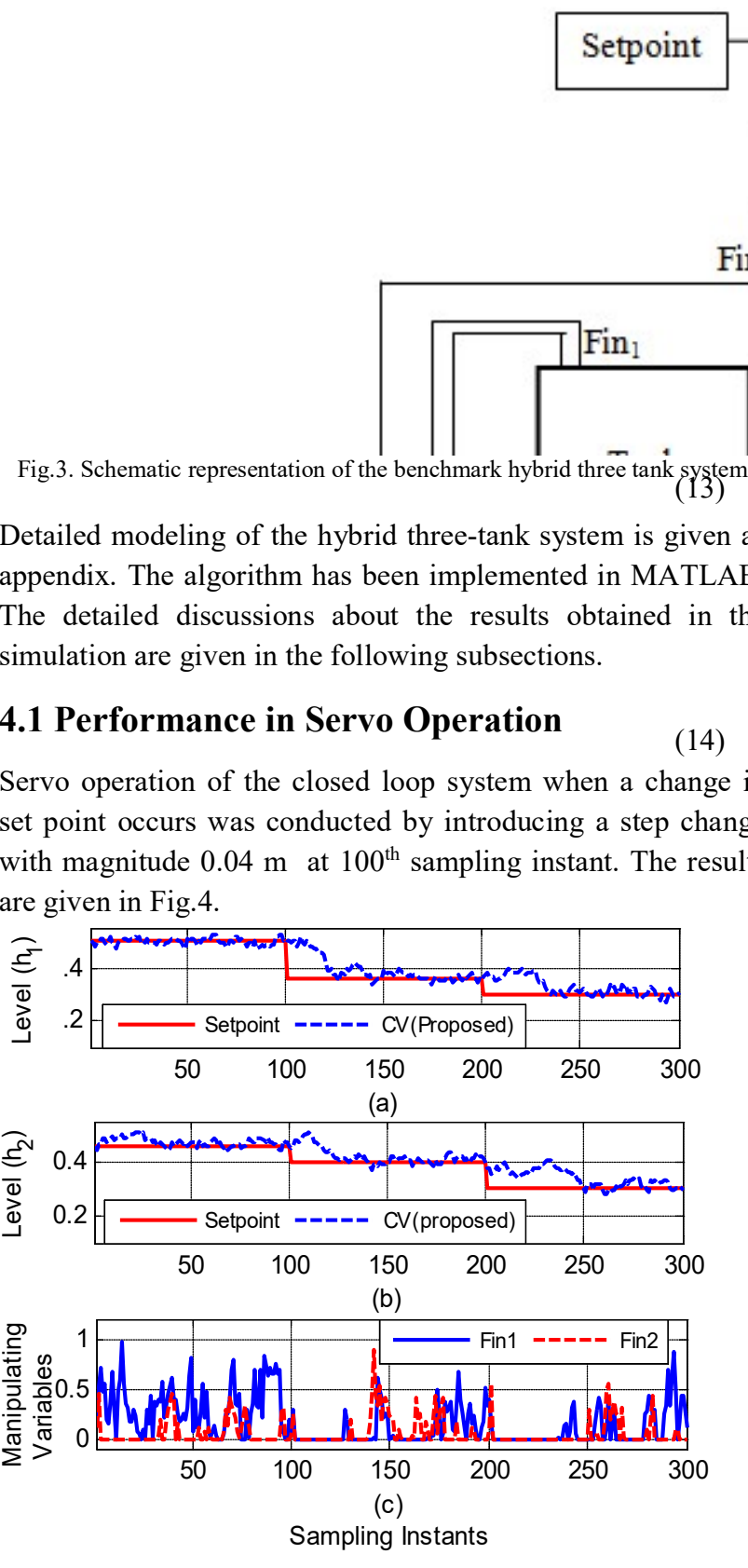

Fig.4. Servo response of hybrid three tank system with ANNC (a) Level in Tank 1, (b) Level in tank 2 (c) Manipulating variables 
Comparison with the best existing related work is shown in Table 2. Comparison of the proposed approach to UKF based approach based on ISE shows that the new approach is better as ISE reduced from 3.3278 to 0.5533 in level, $h_{l}$ and from 3.7203 to 0.4568 in level, $h_{2}$. Also, the average computation time per iteration reduced from 60.65 seconds to 0.0777 seconds. Evolution of true and estimated states of hybrid three-tank system with ANNC (Servo operation) is shown in Fig.5. Evolution of true and estimated values of discrete variables of hybrid three-tank system with ANNC (Servo Operation) is shown in Fig.6.
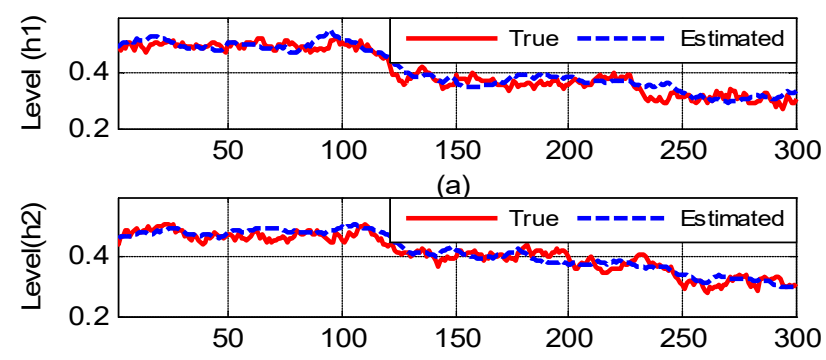

(b)

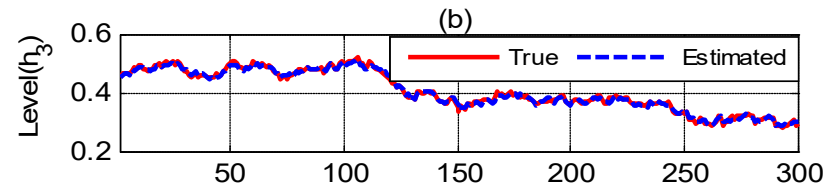

(c)

Sampling Instants

Fig.5. True and estimated states of hybrid three tank system with ANNC (Servo operation) (a) Level in Tank 1, (b) Level in Tank 2, (c) Level in tank 3.
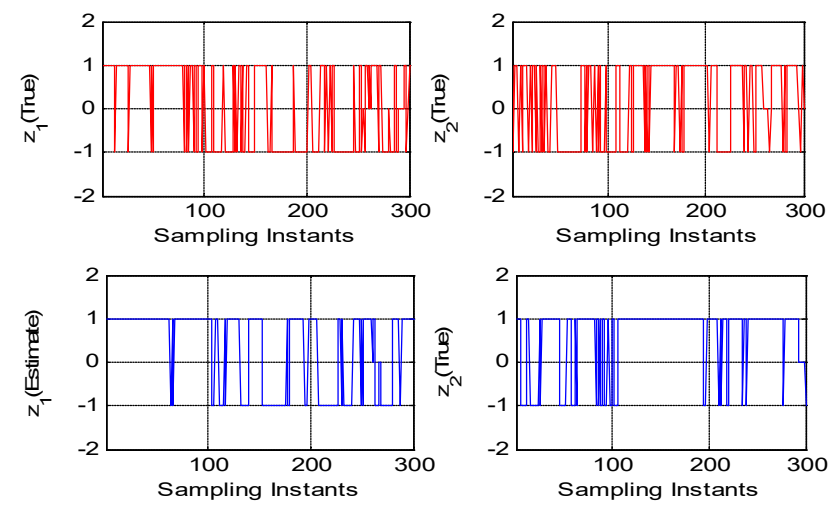

Fig.6. Evolution of true and estimated values of discrete variables of hybrid three tank system with ANNC (Servo Operation)

\subsection{Performance in Regulatory Operation}

The results of the performance of the estimator in regulatory operation are given in Fig.7. Comparison with the best existing related work is shown in Table 3. Comparison of proposed approach to UKF based approach based on standard deviation shows that the new approach is better as standard deviation has reduced from 0.0130 to 0.0127 in level $h_{2}$ and from 0.0484 to 0.0065 in level $h_{3}$ with very close standard deviation in level $h_{l}$. It may be noted that the maximum standard deviation in the case of nonmeasured state variables is 0.0217 in the proposed approach and 0.0484 in the UKF based NMPC $(45 \%$ reduction with proposed approach).

The results of the performance of the controller in regulatory operation are given in Fig.8 and evolution of true and estimated values of discrete variables of hybrid three tank system with ANNC (Regulatory Operation; Disturbance by varying the valve position of fifth hand valve) in Fig.9. Comparison with the best existing related work is shown in Table 4. Comparison of proposed approach to UKF based approach based on ISE shows that the new approach is better as ISE has reduced from 3.5869 to 0.0587 in level $h_{1}$ and from 1.5212 to 0.0366 in level $h_{2}$. Also, the average computation time per iteration has reduced from 59.05 seconds to 0.0739 seconds.

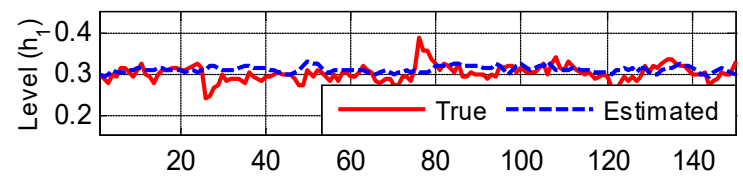

(a)

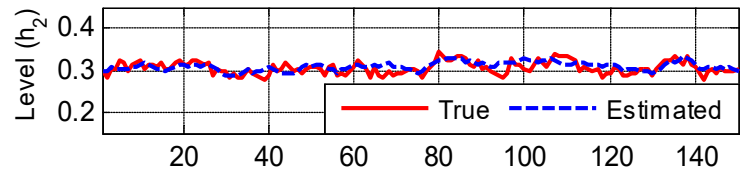

(b)

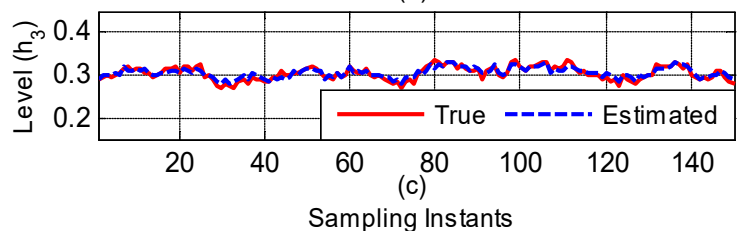

Fig.7. Evolution of true and estimated states of hybrid three tank system with ANNC (a) Level in Tank 1, (b) Level in Tank 2, (c) Level in tank 3
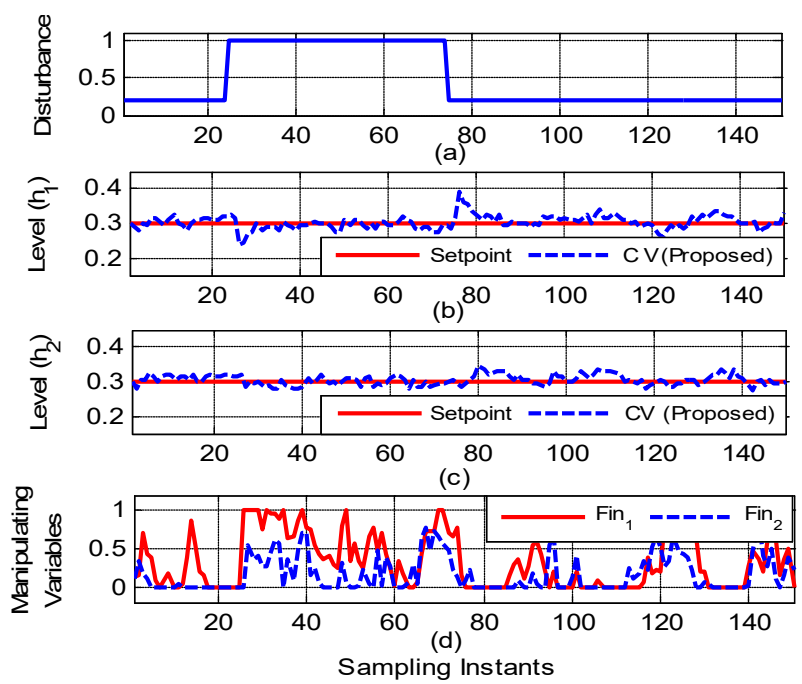

Fig .8. Regulatory response of hybrid three tank system with ANNC (a) disturbance, (b) Level in Tank 1, (c) Level in tank 2 (d) Manipulating variables 

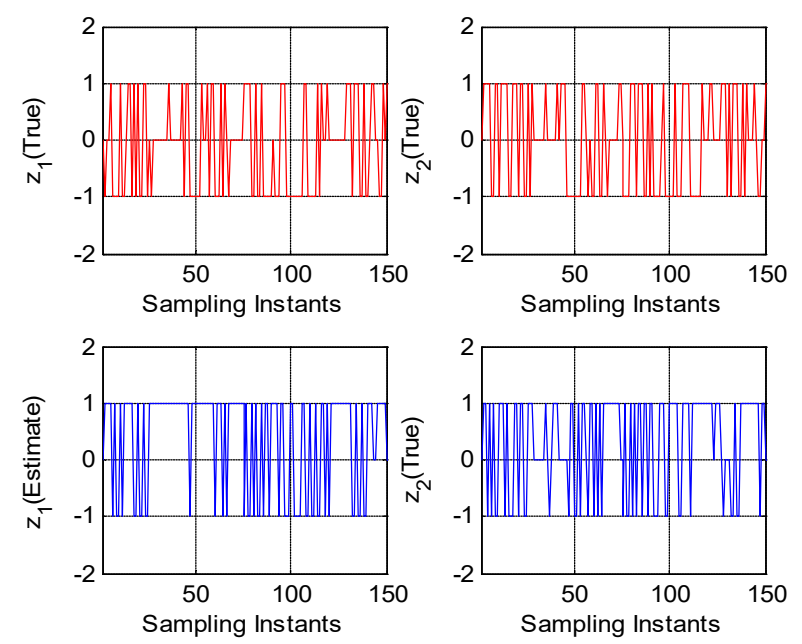

Fig.9. Evolution of true and estimated values of discrete variables of hybrid three tank system with ANNC (Regulatory Operation)

In order to obtain better insight of the ability of the proposed controller to achieve decoupling and offset-free control action, hypothetical situation, in which state and measurement noise are not present, is simulated. Response given in Fig. 10 reveals that the effect in level of tank 2, due to the disturbance in tank 1 is very much less compared with that given in [7]. As in the case of UKF based NMPC in [7], proposed method also giving a slight offset.

Table 3: Regulatory Control Problem: Estimator performance Comparison

\begin{tabular}{lccc}
\hline Controller & $\sigma_{\mathrm{E}}\left(\mathrm{h}_{1}\right)$ & $\sigma_{\mathrm{E}}\left(\mathrm{h}_{2}\right)$ & $\sigma_{\mathrm{E}}\left(\mathrm{h}_{3}\right)$ \\
\hline Proposed & 0.0217 & 0.0127 & 0.0065 \\
UKF based NMPC[7] & 0.0213 & 0.0130 & 0.0484 \\
UKF based NMPC[7] & 0.0242 & 0.0141 & 0.0488 \\
\hline
\end{tabular}

Table 4: Regulatory Control Problem: Controller Performance Comparison

\begin{tabular}{lccc}
\hline Controller & ISE $\left(\mathrm{h}_{1}\right)$ & $\operatorname{ISE}\left(\mathrm{h}_{2}\right)$ & $\begin{array}{c}\text { Avg. Computation } \\
\text { time per iteration } \\
(\mathrm{S})\end{array}$ \\
\hline $\begin{array}{l}\text { Proposed } \\
\text { UKF based }\end{array}$ & 0.0587 & 0.0366 & 0.0739 \\
$\begin{array}{l}\text { NMPC[7] } \\
\text { EnKF based }\end{array}$ & 3.5869 & 1.5212 & 59.05 \\
NMPC[7] & 3.3928 & 1.4011 & 206.44 \\
\hline
\end{tabular}
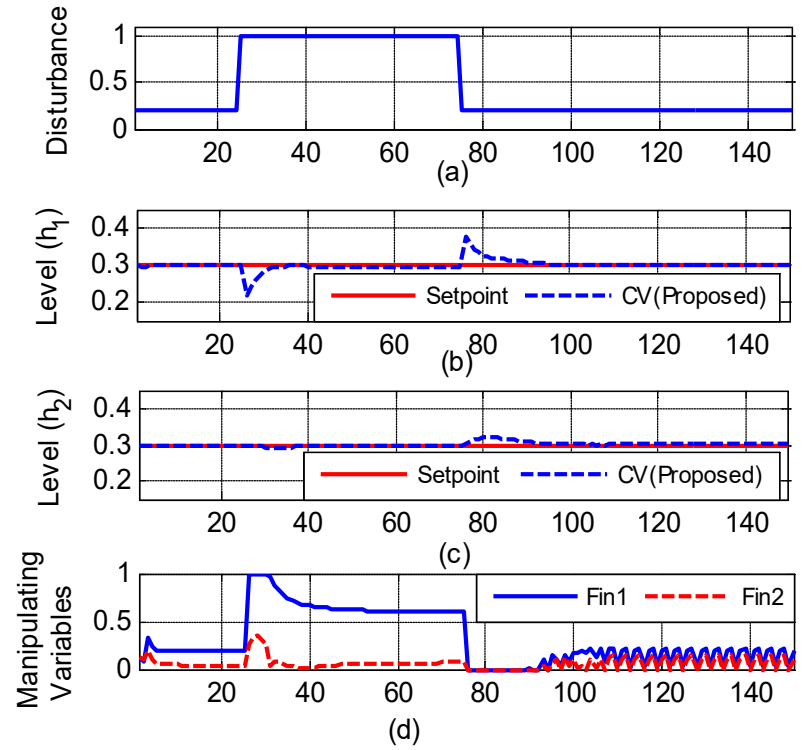

Sampling Instants

Fig .10. Regulatory response of hybrid three tank system with ANNC (without state and measurement noise) (a) disturbance, (b) Level in Tank 1, (c) Level in tank 2 (d) Manipulating variables

\subsection{Plant Model Parameter Mismatch}

The performance of the controller in case of plant model parameter mismatch is considered and the performance is given in Fig. 11. From Table.5, it can be seen that the ISE is improved to 0.0276 from 0.9231 for level $h_{1}$ and to 0.0228 from 1.0579 for level $h_{2}$ when compared to best existing related work based on UKF.

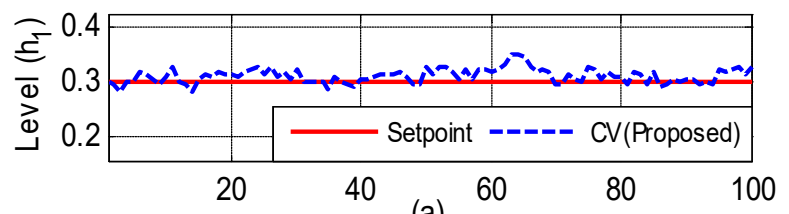

(a)
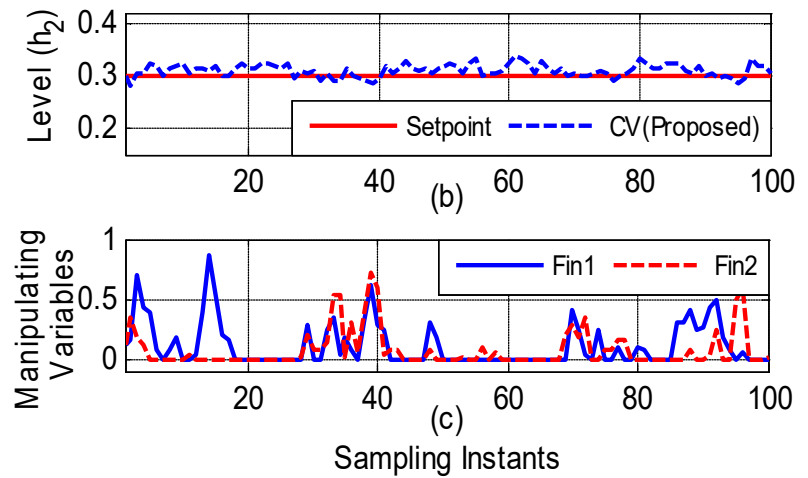

Fig.11. Response of hybrid three tank system with ANNC (Plant-Model mismatch ) (a) Level in Tank 1, (b) Level in Tank 2, (c) Level in tank 3 
Table 5: Plant Model Parameter Mismatch: Controller Performance Comparison

\begin{tabular}{lcc}
\hline Controller & $\operatorname{ISE}\left(\mathrm{h}_{1}\right)$ & $\operatorname{ISE}\left(\mathrm{h}_{2}\right)$ \\
\hline Proposed & 0.0276 & 0.0228 \\
UKF based NMPC [7] & 0.9231 & 1.0579 \\
EnKF based NMPC[7] & 0.8647 & 0.9688 \\
\hline
\end{tabular}

\section{Experimental Results and Performance Analysis}

Real-time experimental validations were carried out on the experimental setup. In addition to the experimental setup, other tools used, which were for the real time implementation are the software Lab VIEW and the NI DAQ (USB6251). In the real system, the performance of the controller in regulatory operation and servo operation based on ISE and average computation time per iteration is shown in Fig. 12, Table 6, Fig. 13, and Table 7. In Table 8 and Fig. 14 response of the system in initial condition mismatch is shown. The response of the system in $+10 \%$ and $-10 \%$ plant model parameter mismatch is given in Tables 9 and 10 and Figures 15 and 16 respectively. Results of hand valve faults which can occur in real time application are given in Table 11, Fig. 17, Table 12 and Fig. 18. The real time experimental results support the simulation results on performance.

Table 6: Regulatory Control Problem: Controller Performance Comparison

\begin{tabular}{cccc}
\hline Controller & ISE $\left(\mathrm{h}_{1}\right)$ & $\mathrm{ISE}\left(\mathrm{h}_{2}\right)$ & $\begin{array}{l}\text { Avg. Computation } \\
\text { time per iteration }(\mathrm{S})\end{array}$ \\
\hline Proposed & 0.0200 & 0.0193 & 0.1038 \\
\hline
\end{tabular}

Table 7: Servo Control Problem: Controller Performance Comparison

\begin{tabular}{lccc}
\hline Controller & ISE( $\left.\mathrm{h}_{1}\right)$ & ISE( $\left(\mathrm{h}_{2}\right)$ & $\begin{array}{l}\text { Avg. Computation } \\
\text { time per iteration }(\mathrm{S})\end{array}$ \\
\hline Proposed & 0.0172 & 0.0144 & 0.1152 \\
\hline
\end{tabular}

Table 8: Initial Condition Mismatch: Controller Performance Comparison

\begin{tabular}{cccc}
\hline Controller ISE $\left(\mathrm{h}_{1}\right)$ & $\mathrm{ISE}\left(\mathrm{h}_{2}\right)$ & $\begin{array}{c}\text { Avg. Computation } \\
\text { time per iteration }(\mathrm{S})\end{array}$ \\
\hline Proposed & 0.0214 & 0.0592 & 0.1017 \\
\hline
\end{tabular}

Table 9: Plant Model Parameter Mismatch (+10\%): Controller Performance Comparison

\begin{tabular}{cccc}
\hline Controller & $\operatorname{ISE}\left(\mathrm{h}_{1}\right)$ & $\operatorname{ISE}\left(\mathrm{h}_{2}\right)$ & $\begin{array}{c}\text { Avg. Computation } \\
\text { time per iteration }(\mathrm{S})\end{array}$ \\
\hline Proposed & 0.0045 & 0.0069 & 0.2112 \\
\hline
\end{tabular}

Table 10: Plant Model Parameter Mismatch (-10\%): Controller Performance Comparison

\begin{tabular}{cccc}
\hline Controller & ISE $\left(\mathrm{h}_{1}\right)$ & $\mathrm{ISE}\left(\mathrm{h}_{2}\right)$ & $\begin{array}{l}\text { Avg. Computation } \\
\text { time per iteration }(\mathrm{S})\end{array}$ \\
\hline Proposed & 0.0122 & 0.0131 & 0.1037 \\
\hline
\end{tabular}

Table 11: Hand Valve Faults -Leakage: Controller Performance Comparison

\begin{tabular}{lccl}
\hline Controller & ISE( $\left.\mathrm{h}_{1}\right)$ & ISE $\left(\mathrm{h}_{2}\right)$ & $\begin{array}{l}\text { Avg. Computation } \\
\text { time per iteration }(\mathrm{S})\end{array}$ \\
\hline Proposed & 0.0600 & 0.0077 & 0.0768 \\
\hline
\end{tabular}

Table 12: Hand Valve Faults -Clogging: Controller Performance Comparison

\begin{tabular}{cccc}
\hline Controller & ISE $\left(\mathrm{h}_{1}\right)$ & $\mathrm{ISE}\left(\mathrm{h}_{2}\right)$ & $\begin{array}{c}\text { Avg. Computation } \\
\text { time per iteration }(\mathrm{S})\end{array}$ \\
\hline Proposed & 0.0082 & 0.0078 & 0.0943 \\
\hline
\end{tabular}

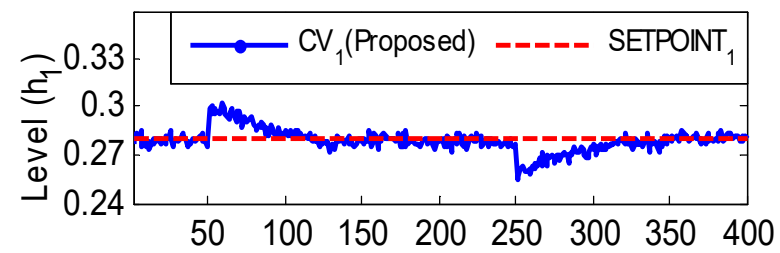

(a)

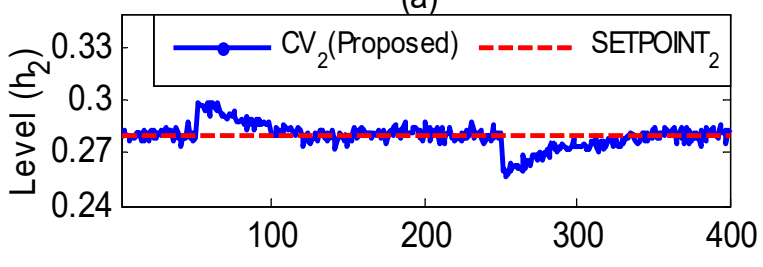

(b)

Sampling Instants

Fig.12. Regulatory response of hybrid three tank system with ANNC (a) Level in Tank 1, (b) Level in tank 2 


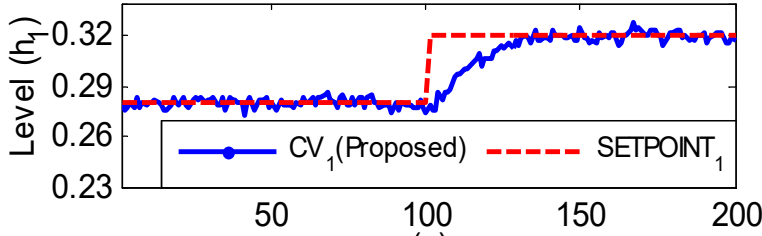

(a)

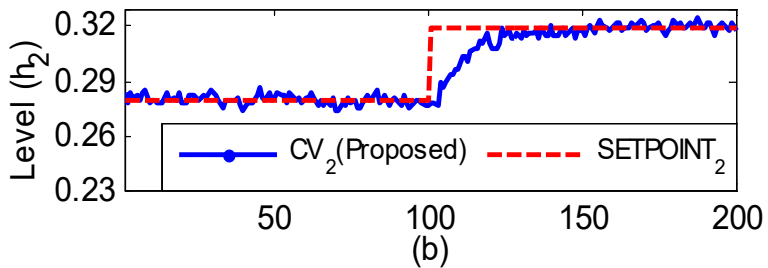

Sampling Instants

Fig.13. Servo response of hybrid three tank system with ANNC (a) Level in Tank 1, (b) Level in tank 2

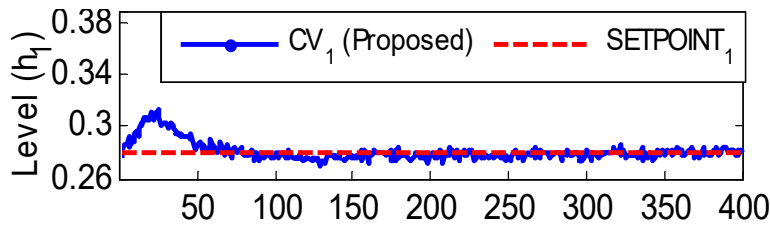

(a)

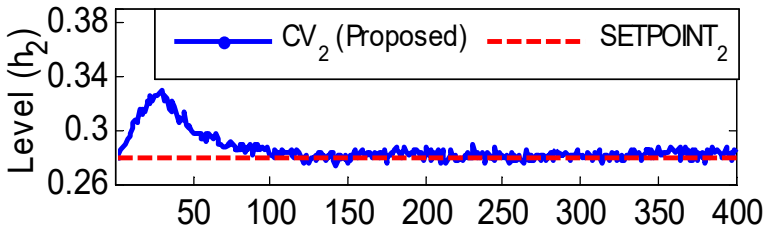

(b)

Sampling Instants

Fig. 14. Closed response of hybrid three tank system with ANNC (Initial Condition Mismatch ) (a) Level in Tank 1, (b) Level in Tank 2
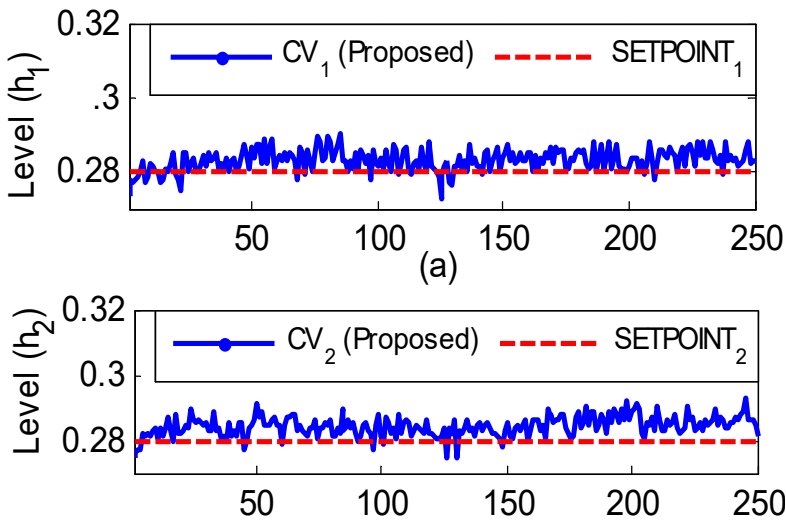

(b)

Sampling Instants

Fig.15. Closed response of hybrid three tank system with ANNC (Plant-Model mismatch $+10 \%$ ) (a) Level in Tank 1, (b) Level in Tank 2

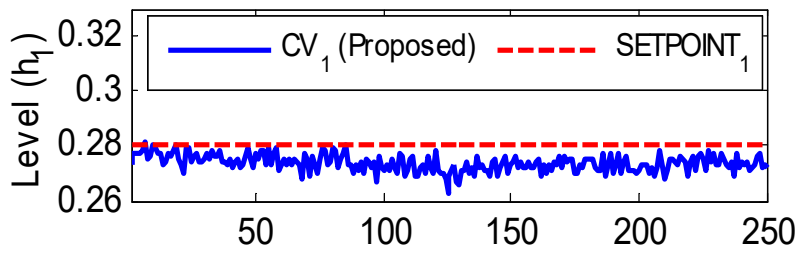

(a)

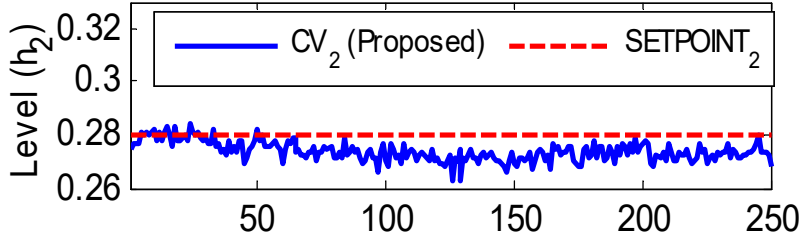

(b)

Sampling Instants

Fig. 16. Closed response of hybrid three tank system with ANNC (Plant-Model mismatch $-10 \%$ ) (a) Level in Tank 1, (b) Level in Tank 2

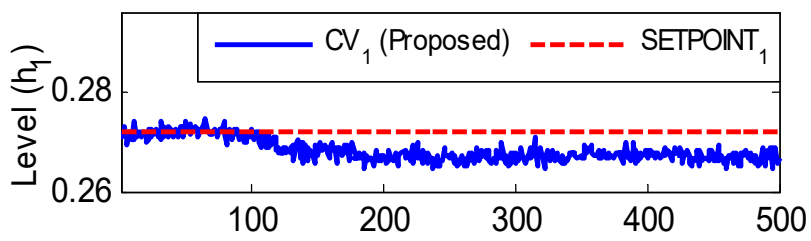

(a)

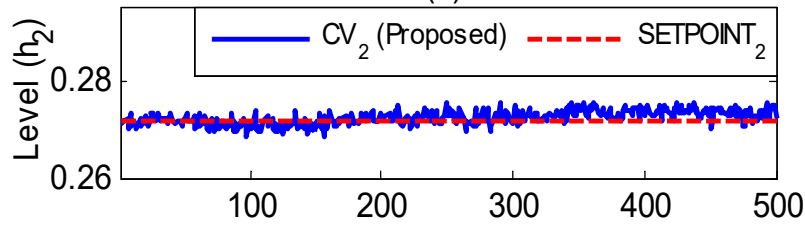

(b)

Sampling Instants

Fig.17. Closed response of hybrid three tank system with ANNC (Handvalve fault-Leakage ) (a) Level in Tank 1, (b) Level in Tank 2
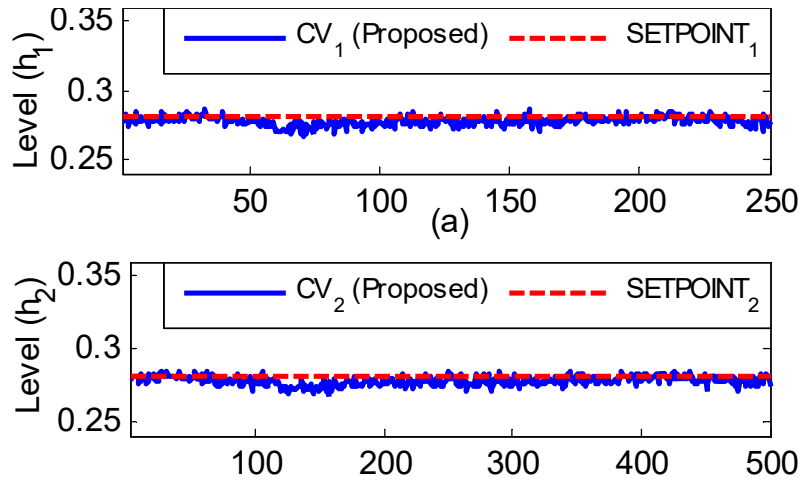

(b)

Sampling Instants

Fig. 18. Closed response of hybrid three tank system with ANNC (Handvalve fault-Clogging ) (a) Level in Tank 1, (b) Level in Tank 2 


\section{Conclusion}

An ANN estimation based control scheme which offers better state estimates and hence better control, with a nonlinear approach in correcting the a priori estimates by avoiding statistical linearization involved in derivative free estimation is proposed. On comparing with best exiting related work based on statistical linearization, $85 \%, 97 \%$ and $97 \%$ reduction in Integral Square Error (ISE), between controlled variable and set point, for servo, regulatory and plant model parameter mismatch operations respectively, and a $45 \%$ reduction in standard deviation $(\sigma)$ of error between true and estimated values of non-measurable states for regulatory control operation were obtained. In addition to these performance improvements, the attracting feature of the proposed method is the time required for the computation of control signals and is very much less than the sampling time of the process which ensures the capability of online implementation as direct control algorithm using the proposed approach. The experimental studies conducted on a real plant illustrate the robust performance of the proposed controller in offering better online control of hybrid dynamic system under real time operating constraints.

\section{References}

[1] Lennartson, M. Tittus, B. Egardt, and S. Pettersson, "Hybrid systems in process control”, IEEE Control Systems, 16,(5), (2002), 45-56.

[2] Goebel R., Sanfelice R. G. Teel, A. R., Hybrid Dynamical Systems , IEEE Control Systems Magazine, 29(2), (2009), 28-93.

[3] Goebel, R; Sanfelice, R, G.and Teel, A.R., Hybrid Dynamical Systems: Modeling, Stability, and Robustness, Princeton University Press, 2012.

[4] Narasimhan, S. and Biswas, G., Model-Based Diagnosis of Hybrid Systems, IEEE Transactions on Systems, Man and Cybernetics, 37(3), (2007), 348-361.

[5] N. L. Ricker, Model predictive control with state estimation, Ind. Eng. Chem. Res.29, (1990), 374-382.

[6] J. H. Lee, N. L. Ricker, Extended Kalman filter based non-linear model predictive control, Ind. Eng. Chem. Res. 33(6), (1994), 1530-1541.

[7] J. Prakash, Sachin C. Patwardhan, Sirish L. Shah, State estimation and nonlinear predictive control of autonomous hybrid system using derivative free state estimators, Journal of Process Control, 20, (2010), 787-799

[8] G. Ferrari Trecate, D. Mignone, Moving horizon estimation for hybrid systems, IEEE Transactions on Automatic Control, 47(10), (2002), 1663-1676.

[9] S. J. Julier, J.K. Uhlmann, Unscented filtering and nonlinear estimation, Proc. IEEE, 92 (3), (2004), 401-422

[10] Sachin C. Patwardhan, Shankar Narasimhan, Prakash Jagadeeshan, Bhushan Gopaluni, Sirish L. Shah, Nonlinear Bayesian state estimation: A review of recent developments, Control Engineering Practice, 20(10), (2012), 933-953

[11] Jean Thomas, Robust Model Predictive Controller for Uncertain Piecewise Affine Systems, Arabian Journal of Science and Engineering, vol. 39, issue 10, pp. 7421- 7432, 2014
[12] J. Prakash, Sachin C. P, S. L. Shah, Control of an Autonomous Hybrid System Using a Nonlinear Model Predictive Controller, In Proc. 17th World Congress of IFAC, (2008), 12510-12515.

[13] Jagadeeshan P., Sachin C. P., Sirish L. S, Design and Implementation Fault Tolerant Model Predictive Control Scheme on a Simulated Model of Three-Tank Hybrid System, Conference on Control and Fault Tolerant Systems, France, (2010), 173-178.

[14] Lefebvre, T.; Bruyninckx, H.; De Schutter, J., Comment on "A new method for the nonlinear transformation of means and covariances in filters and estimators", IEEE Transactions on Automatic Control, 47(.8), (2002), 1406-1409.

[15] Kumar, S., Prakash, J. and Kanagasabapathy, P. “A critical evaluation and experimental verification of Extended Kalman Filter, Unscented Kalman Filter and Neural State Filter for state estimation of three phase induction motor", Applied Soft Computing, Vol 11, Issue 3, pp. 3199 3208,2011

[16] K. Zhang, Yuan F, Guo, J.Wang G., A Novel Neural Network Approach to Transformer Fault Diagnosis Based on MomentumEmbedded BP Neural Network Optimized by Genetic Algorithm and Fuzzy C Means, Arabian Journal of science and Engineering, vol. 41, issue 9, pp. 3451-3461, 2016

[17] Rajeev Kumar D., K. Singh, R. Kumar, S. Upadhaya, Simulationbased Artificial Neural Network Predictive Control of BTX Dividing Wall Column, Arabian Journal of science and Engineering, vol. 40, issue 12, pp. 3393-3407, 2015

[18] G. Parlos, S. K. Menon, A. F. Atiya, An Algorithmic Approach to Adaptive State Filtering Using Recurrent Neural Networks, IEEE transactions on neural networks, 12(6), (2001), 1411-1432.

[19] N. N. Nandola, S. Bharatiya, Hybrid system identification using a structural approach and its model based control: An experimental validation, Nonlinear Analysis: Hybrid Systems, 3(2), (2009), 87-100.

[20] Lane, S, H and Stengel, R, F., Flight Control Design Using Non linear Inverse Dynamics, Automatica, 24(4), (1988), 471-483.

[21] Gorazd Karer, Gasper Music, Igor Skrjanc, Borut Zupancic, Feedforward control of a class of hybrid systems using an inverse model, Mathematics and Computers in Simulation, 82(3), (2011), 414-427.

[22] Spong, M.W and Ortega, R.; De Schutter, J., On Adaptive Inverse Dynamics Control of Rigid Robots , IEEE Transactions on Automatic Control, 35(1), (1990), 92-95.

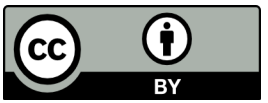

(C) 2020 by the authors. Submitted for possible open access publication under the terms and conditions of the Creative Commons $\left.\begin{array}{llll}\text { Attribution } & (\mathrm{CC} & \mathrm{BY}\end{array}\right)$ license (http://creativecommons.org/licenses/by/4.0/). 